\title{
DETERMINATION OF SUBMONOLAYER COVERAGES USING AUGER ESCAPE DEPTHS
}

\author{
P.J. GoDowski \\ W. Trzebiatowski Institute of Low Temperature and Structure Research \\ Polish Academy of Sciences \\ P.O. Box 937, 50-950 Wroclaw, Poland \\ AND E.L. HARDEGREE \\ Department of Chemistry, Abilene Christian University, Abilene, TX 79699, USA
}

(Received December 22, 1993)

\begin{abstract}
A simple method for determining submonolayer adsorbate coverages by means of Auger electron spectroscopy is presented. The procedure is based on the ratio of low to high kinetic energy Auger signals from the substrate alone, therefore it does not require a direct measurement of adsorbate peaks. It may be of special interest in cases where the adsorbate signal is difficult or impossible to measure, as well as when adsorbate and substrate peaks overlap or when peaks are absent ( $\mathrm{H}$ or $\mathrm{He}$ ) or of low intensity. The method is tested by quantification of sulphur layer on the $\mathrm{Ni}(001)$ and on the polycrystalline iron surface.
\end{abstract}

PACS numbers: 79.20.Fv, 68.10.Jy, 82.65.My

It is common in adsorption studies to determine adsorbate surface coverages by use of Auger electron spectroscopy (AES). Sulfur uptake on nickel, for example, has often been characterized by this method [1-3]. To convert raw Auger signals to coverage, some type of calibration is necessary. Calibration may be done with radioactive tracer technique [1], by correlation of Auger peak-to-peak ( $p-p)$ heights with LEED (Low Energy Electron Diffraction) patterns [4-8] or with a quartz oscillator microbalance and by mass spectrometry [9]. Quantitative Auger electron analysis is not simple for "bulk" samples, especially in the case where the concentration varies with depth (e.g. [10]). During epitaxial growth, the Auger electron signal of the substrate decreases from bulk value to zero as a polygonal curve inscribed into a decaying exponential [11]. A similar picture holds for the Auger signal of adsorbate, but the polygonal curve increases from zero toward a constant value. If interdiffusion between adsorbate and substrate and/or clustering of the overlayer atoms are important, the situation is more difficult and a rounding of the polygonal curve is expected to occur [11]. The variation of Auger electron 
intensities with increasing film thickness could be considered to characterize the mode of growth (e.g. [12-17]). Due to the above-mentioned difficulties, the best approximation in determination of thin film thickness (at least several layers or equivalent quantity of adsorbate) seems the use of exponential dependence as was shown by Smith and Southworth [18]. Quantitative AES is simple when the atoms to be studied are distributed only in two dimensions (in the plane above the substrate, i.e. without intermixed region) as in adsorption systems with submonolayer adsorbate coverages. General nature of the linear relationship between $p-p$ height, $h$, and coverage, $\Theta(0 \leq \Theta \leq 1)$, was confirmed experimentally many times (e.g. [19-27]). The appropriate formulae may be written as

$$
\begin{aligned}
& h_{\mathrm{A}}(\Theta)=h_{\mathrm{A}}^{1} \Theta=h_{\mathrm{A}}^{\mathrm{B}}\left[1-\exp \left(-1 / D_{\mathrm{A}}^{\mathrm{A}}\right)\right] \Theta=h_{\mathrm{A}}^{\mathrm{B}} K_{\mathrm{A}} \Theta, \\
& h_{\mathrm{S}}(\Theta)=h_{\mathrm{S}}^{\mathrm{B}}-\left[h_{\mathrm{S}}^{\mathrm{B}}-h_{\mathrm{S}}^{\mathrm{B}} \exp \left(-1 / D_{\mathrm{S}}^{\mathrm{A}}\right)\right] \Theta \\
& \quad=h_{\mathrm{S}}^{\mathrm{B}}\left\{1-\left[1-\exp \left(-1 / D_{\mathrm{S}}^{\mathrm{A}}\right)\right] \Theta\right\}=h_{\mathrm{S}}^{\mathrm{B}}\left(1-K_{\mathrm{S}} \Theta\right) .
\end{aligned}
$$

The various terms in these equations are defined below. $h_{\mathrm{A}}(\Theta)$ and $h_{\mathrm{S}}(\Theta)$ are Auger $\mathrm{p}-\mathrm{p}$ heights from the adsorbate (A) or substrate $(\mathrm{S})$ at an adsorbate coverage $\Theta$, $h_{\mathrm{A}}^{1}$ being the adsorbate signal for $\Theta=1 . h_{\mathrm{A}}^{\mathrm{B}}$ and $h_{\mathrm{S}}^{\mathrm{B}}$ are Auger $\mathrm{p}$-p heights for pure, bulk (superscript B) adsorbate (subscript A) or substrate (subscript $S$ ). $D_{A}^{A}$ and $D_{\mathrm{S}}^{\mathrm{A}}$ are attenuation lengths of adsorbate (subscript $\mathrm{A}$ ) or substrate (subscript $\mathrm{S}$ ) Auger electrons through the adsorbate (superscript A) overlayer, expressed in monolayers. Their values depend on the kinetic energy of the electrons. $K_{\mathrm{A}}$ and $K_{\mathrm{S}}$ are constants for a given kinetic energy, depending on $D_{i}^{\mathrm{A}}(i=\mathrm{A}, \mathrm{S})$ only.

The above equations are almost self-evident; in the relation (1) Auger signal is proportional to the number of adsorbed atoms via $\Theta$. In the relation (2) the fraction $(1-\Theta)$ of the surface is uncovered by the adsorbate and contributes fully (without attenuation term) to the substrate Auger signal. The substrate signal due to the covered fraction of the surface, $\theta$, is attenuated in passing through the adsorbate, hence the $\exp (-1 / D)$ term. The attenuation length $D$ is in relation with the electron escape depth $\lambda$ through the dependence on the geometry of the electron energy analyzer. For both retarding field (RFA) and cylindrical mirror (CMA) analyzers the relationship is given by $D(E)=0.74 \lambda(E)$, where $\lambda(E)$ and thus $D(E)$ depends on electron kinetic energy $E[20]$. For the range of kinetic energies commonly used in AES, the electron escape depth increases with $E$, giving a greater depth for high energy than for low energy Auger electrons. Adsorbate atoms thus attenuate intensity of substrate low energy peaks with coverage more quickly than intensity of high energy ones. Figure 1 shows the effect of surface coverage on the normalized substrate Auger $\mathrm{p}-\mathrm{p}$ height, $h_{\mathrm{S}} / h_{\mathrm{S}}^{\mathrm{B}}\left(=1-K_{\mathrm{S}} \Theta\right)$, for various energies of Auger electrons (the parameter of the line).

To help obviate errors arising from accidental changes in experimental conditions, in quantification many workers use the p-p heights ratio of the adsorbate to the substrate. As may be seen from Eqs. (1) and (2), this ratio is not linear with coverage $[22,25]$ (except at very low coverages, where $1-K_{\mathrm{S}} \Theta \approx 1$ can be assumed). In this note we present the method of adsorbate coverage determination basing on Eq. (2) only. The method is similar to the one presented by Smith and 


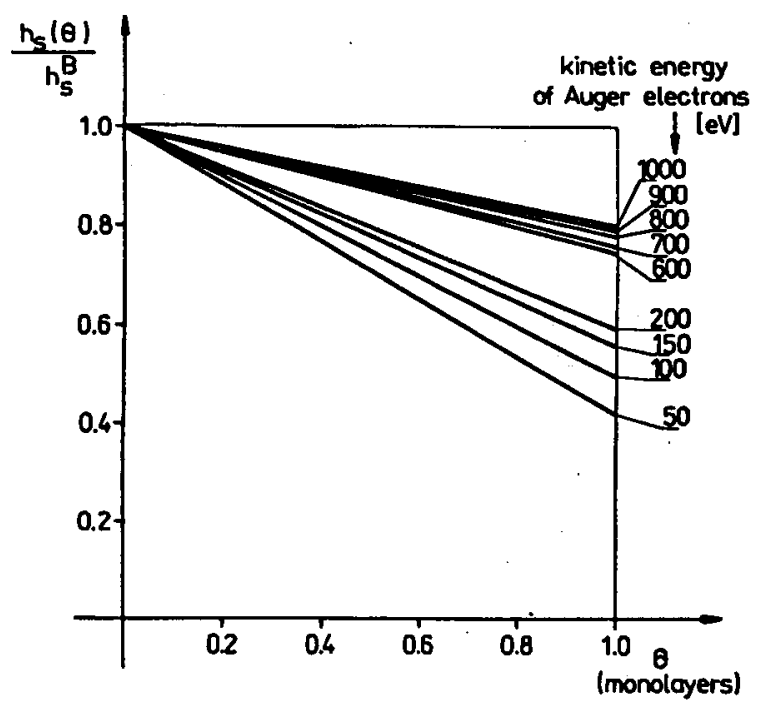

Fig. 1. Dependence of normalized substrate Auger signal, $h_{\mathrm{S}} / h_{\mathrm{S}}^{\mathrm{B}}$, on coverage $\Theta(0 \leq \Theta \leq 1)$. For various kinetic energies of Auger electrons, straight lines are drawn according to relation (2) using formula ( $5 b$ ) for $\lambda$ (see text). There exists serious difference between line slopes corresponding to high $(600 \div 1000 \mathrm{eV})$ and low energy $(50 \div 200 \mathrm{eV})$ Auger peaks. Attenuation of the high energy electrons with the adsorbate coverage is not negligible in quantitative AES.

Southworth [18], but it applies to submonolayer coverages, i.e., up to one monolayer. For $0 \leq \Theta \leq 1$ the Auger yield of substrate transition decays linearly with $\Theta$, not exponentially with layer thickness and as a matter of fact the exponential line is an approximation of that straight line (not vice versa) and this approximation cannot be longer valid in submonolayer region. The method does not require a measurement of the adsorbate Auger signal, and therefore it avoids the adsorbate signal calibration, that is, determination of $h_{\mathrm{A}}^{1}$ (or $h_{\mathrm{A}}$ for some other known a priori coverage). However, knowledge of $\lambda$ (and thus $D_{i}^{\mathrm{A}}$ ) values is the important alternative condition. Although $\Theta$ may be found from Eq. (2) by measuring the $\mathrm{p}$-p height of a single peak for both clean $\left(h_{\mathrm{S}}^{\mathrm{B}}\right)$ and covered $\left(h_{\mathrm{S}}\right)$ substrate, this does not adequately allow for errors due to changes in experimental conditions while going from the clean to the dosed spectra, since it employs two absolute $p-p$ heights, each from a different spectrum. Differences in excitation beam current, for example, could seriously affect the absolute peak heights for the two spectra. A better method is to employ the p-p height ratios for each spectrum. If the substrate has two Auger transitions: low and high energy ones, the p-p height ratios of low to high energy substrate peaks for clean and covered surface can be registered. From Eq. (2), denoting by $\mathrm{L}-$ low and $\mathrm{H}$ - high kinetic energy transitions, we have

$$
\frac{h_{\mathrm{L}}}{h_{\mathrm{H}}}(\Theta)=\frac{h_{\mathrm{L}}^{\mathrm{B}}}{h_{\mathrm{H}}^{\mathrm{B}}} \frac{1-\left[1-\exp \left(-1 / D_{\mathrm{L}}\right)\right] \Theta}{1-\left[1-\exp \left(-1 / D_{\mathrm{H}}\right)\right] \Theta}=\frac{h_{\mathrm{L}}^{\mathrm{B}}}{h_{\mathrm{H}}^{\mathrm{B}}} \frac{1-K_{\mathrm{L}} \Theta}{1-K_{\mathrm{H}} \Theta} .
$$


Defining $r_{\mathrm{LH}}:=h_{\mathrm{L}} / h_{\mathrm{H}}$ and $r_{\mathrm{LH}}^{\mathrm{B}}:=h_{\mathrm{L}}^{\mathrm{B}} / h_{\mathrm{H}}^{\mathrm{B}}$ we obtain:

$$
\Theta=\frac{r_{\mathrm{LH}}^{\mathrm{B}}-r_{\mathrm{LH}}}{r_{\mathrm{LH}}^{\mathrm{B}}\left[1-\exp \left(-1 / D_{\mathrm{L}}\right)\right]-r_{\mathrm{LH}}\left[1-\exp \left(-1 / D_{\mathrm{H}}\right)\right]}=\frac{r_{\mathrm{LH}}^{\mathrm{B}}-r_{\mathrm{LH}}}{r_{\mathrm{LH}}^{\mathrm{B}} K_{\mathrm{L}}-r_{\mathrm{LH}} K_{\mathrm{H}}} .
$$

Since the Auger spectrum of the adsorbate is not required, Eq. (4) can be applied to cases where the adsorbate signal is inaccessible. Such an event is met in the case of sulfur-molybdenum system, where the $S$ peak is obscured by the overlapping Mo peak. Equation (4) should be applicable as well in determination of coverages of adsorbed $\mathrm{H}$ or $\mathrm{He}$ for which as its intrinsic property there is not any Auger effect.

The effectiveness of the method can be checked by using Auger data for sulfur layers reported in the literature. The electron escape depth in solids obeys a universal curve [28] which is described by the expression as

$$
\lambda=538 / E^{2}+0.41(a E)^{1 / 2}, \mathrm{ML}
$$

where $a$ is the monolayer thickness in nanometers, $E$ is in $\mathrm{eV}$, and $\lambda$ is in monolayers (ML). Upon inserting $a=0.212 \mathrm{~nm}$ which corresponds to the covalent diameter of sulfur, we obtain $\lambda$ for any Auger electrons travelling through sulfur layer

$$
\lambda=538 / E^{2}+0.189 E^{1 / 2}, \text { ML. }
$$

As the first example the Auger data for sulfur adsorbed on $\mathrm{Ni}(001)[3,25]$ were taken. Introducing $D$ values $(D=0.74 \lambda)$ into Eq. (4) as $K$ 's [20], the $\Theta$ 's were calculated and collected with the other appropriate data in Table $I$. The coverages

\section{TABLE I}

Quantitative results for $\mathrm{S} / \mathrm{Ni}(001)$. Parameters used for calculation are the following: kinetic energies of Auger electrons: $E_{\mathrm{L}}=61 \mathrm{eV}, E_{\mathrm{H}}=848 \mathrm{eV}$ and attenuation lengths obtained by Eq. (5b): $D_{\mathrm{L}}=1.20 \mathrm{ML}, D_{\mathrm{H}}=4.07 \mathrm{ML}$.

\begin{tabular}{l|c|c|c|c|c}
\hline \hline Analyzer & \multirow{2}{*}{$r_{\mathrm{LH}}^{\mathrm{B}}$} & $r_{\mathrm{LH}}(\Theta)$ & \multicolumn{3}{|c}{$\Theta$} \\
\cline { 4 - 6 } & & & Eq. (4) & Calibration & Ref. \\
\hline Double-pass & & & & & \\
CMA & 1.53 & 1.24 & 0.49 & 0.50 & {$[3]$} \\
\hline RFA & 38.0 & 30.5 & 0.51 & 0.50 & {$[25]$} \\
& 38.0 & 34.5 & 0.25 & 0.25 & {$[25]$}
\end{tabular}

are in good agreement with the ones determined by conventional methods employing calibration of the Auger adsorbate signal with the good ordered superstructure of LEED pattern. The second example refers to the sulfur layer obtained by segregation process. During competitive segregation of arsenic and sulfur on polycrystalline iron surface, in thermal equilibrium at temperatures above $1070 \mathrm{~K}$, the surface is covered only by sulfur $[29,30]$. Using the ratio of low energy $\mathrm{Fe} 45 \mathrm{eV}$ to high energy $\mathrm{Fe} 651 \mathrm{eV}$ Auger peak, the sulfur surface coverage was determined as $\Theta=0.40$. This value is close to the saturation value in the sulfur-iron system 
TABLE II

Values of the $p-p$ ratios of the Auger transitions and corresponding surface concentration of sulfur on polycrystalline iron. The attenuation lengths were obtained using Eq. (5b).

\begin{tabular}{l|c|c|c|c|c}
\hline \hline Analyzer & $r_{\mathrm{LH}}^{\mathrm{B}}$ & $r_{\mathrm{LH}}(\Theta)$ & \multicolumn{3}{|c}{$\Theta$} \\
\cline { 4 - 6 } & & & Eq. (4) & Calibration & Ref. \\
\hline CMA & 1.76 & 1.50 & 0.40 & 0.41 & {$[31]$}
\end{tabular}

in that temperature $\Theta=0.41$ found by Grimal and Marcus [31] using X-ray photoelectron spectroscopy (Table II). In both cases, the $\Theta$ express the coverage in terms of surface density of substrate monolayer, i.e. for $\mathrm{Ni}(001)$ and for polycrystalline Fe the values of $1.61 \times 10^{15}$ and $1.92 \times 10^{15}$ at. $\mathrm{cm}^{-2}$, respectively, were taken.

The Auger signal can be measured with high accuracy, e.g., when the signal is recorded several times for statistics or is continuously monitored (many points) during adsorption. The precision in $h$ values measurements in such cases cannot be greater than $\pm 0.5 \%$ and the coverage, $\Theta$, is determined to the precision of $\pm 2 \%$. The method could be applied for very stable AES systems and the measurements should be taken with a carefully considered strategy [32].

\section{References}

[1] M. Perdereau, Surf. Sci. 24, 239 (1971).

[2] E.L. Hardegree, Pin HO, J.M. White, Surf. Sci. 165, 488 (1986).

[3] E.L. Hardegree, J.M. White, Surf. Sci. 175, 78 (1986).

[4] J.E. Demuth, T.N. Rhodin, Surf. Sci. 45, 249 (1974).

[5] R. Ramanathan, J.M. Blakely, Mater. Lett. 2, 12 (1983).

[6] P.C. Wong, M.Y. Zhou, K.C. Hui, K.A.R. Mitchell, Surf. Sci. 163, 172 (1985).

[7] S.W. Jorgensen, R.J. Madix, Surf. Sci. 163, 19 (1985).

[8] J.N. Andersen, Surf. Sci. 192, 583 (1987).

[9] E. Bauer, in: Interactions on Metal Surfaces, Ed. R. Gomer, Springer-Verlag, Berlin 1975, p. 227.

[10] C.J. Powell, M.P. Seah, J. Vac. Sci. Technol. A 8, 735 (1990).

[11] R. Germar, W. Durr, J.W. Krewer, D. Pescia, W. Gudat, Appl. Phys. A 47, 393 (1988).

[12] R. Siuda, Surf. Sci. 140, 19 (1984).

[13] F.C.M.J.M. Van Delft, A.D. Van Langeveld, B.E. Nieuwenhuys, Surf. Sci. 152/153, 270 (1985).

[14] F.C.M.J.M. Van Delft, A.D. Van Langeveld, B.E. Nieuwenhuys, Thin Solid Films 123, 333 (1985).

[15] S. Ossicini, R. Memeo, F. Ciccacci, J. Vac. Sci. Technol. A 3, 387 (1985).

[16] M. Ece, R.W. Vook, J. Vac. Sci. Technol. A 6, 1036 (1988). 
[17] O. Milo, A. Many, Y. Goldstein, J. Vac. Sci. Technol. A 7, 2688 (1989).

[18] J.F. Smith, H.N. Southworth, Surf. Sci. 122, L619 (1982).

[19] D.C. Jackson, T.E. Gallon, A. Chambers, Surf. Sci. 36, 381 (1973).

[20] M.P. Seah, Surf. Sci. 40, 595 (1973).

[21] E.N. Sickafus, J. Vac. Sci. Technol. 11, 299 (1974).

[22] C. Argile, G.E. Rhead, Surf. Sci. 53, 659 (1975).

[23] J.P. Biberian, G.A. Somorjai, Appl. Surf. Sci. 2, 352 (1979).

[24] M.P. Seah, J. Vac. Sci. Technol. 17, 16 (1980).

[25] P.J. Godowski, Acta Univ. Wratisl. 847, 87 (1985).

[26] R. Siuda, Surf. Sci. 177, L1011 (1986).

[27] M.P. Seah, Vacuum 36, 399 (1986).

[28] M.P. Seah, W.A. Dench, Surf. Interface Anal. 1, 2 (1979).

[29] P.J. Godowski, D. Costa, P. Marcus, in preparation.

[30] D. Costa, A. Carraretto, P.J. Godowski, P. Marcus, J. Mat. Sci. Lett. 12, 135 (1993).

[31] J.M. Grimal, P. Marcus, Surf. Sci. 249, 171 (1991).

[32] C.J. Powell, M.P. Seah, J. Vac. Sci. Technol. A 8, 735 (1990). 